ITEP-YM-7-92

August, 1992

\title{
Adjoint Fermions Induce QCD
}

\author{
S. Khokhlachev \\ Cybernetics Council, Academy of Science \\ Vavilov st. 40, 117333 Moscow, Russia \\ and \\ Yu. Makeenko円 \\ Institute of Theoretical and Experimental Physics \\ B.Cheremuskinskaya 25, 117259 Moscow, Russia
}

\begin{abstract}
We propose to induce QCD by fermions in the adjoint representation of the gauge group $S U\left(N_{c}\right)$ on the lattice. We consider various types of lattice fermions: chiral, Kogut-Susskind and Wilson ones. Using the mean field method we show that a first order large- $N$ phase transition occurs with decreasing fermion mass. We conclude, therefore, that adjoint fermions induce QCD. We draw the same conclusion for the adjoint scalar or fermion models at large number of flavors $N_{f}$ when they induce a single-plaquette lattice gauge theory. We find an exact strong coupling solution for the adjoint fermion model and show it is quite similar to that for the KazakovMigdal model with the quadratic potential. We discuss the possibility for the adjoint fermion model to be solvable as $N_{c} \rightarrow \infty$ in the weak coupling region where the Wilson loops obey normal area law.
\end{abstract}

\footnotetext{
${ }^{1}$ E-mail: makeenko@vxitep.itep.msk.su / makeenko@desyvax.bitnet / makeenko@nbivax.nbi.dk
} 


\section{Introduction}

This paper is inspired by a recent work by Kazakov and Migdal [1] who proposed to induce QCD by a scalar field on the lattice. This scalar field is taken in the adjoint representation of the gauge group $S U\left(N_{c}\right)$ while the gauge field is attached in the usual way to make the model gauge invariant except no kinetic term for the gauge field. This construction makes the model solvable in the large- $N_{c}$ limit. The latter circumstance differs the Kazakov-Migdal model from the previous attempts to induce Wilson lattice gauge theory by fundamental-representation scalars [2] or fermions [3] at the number of flavors (or different species) $N_{F} \sim N_{c}$.

A price for the solubility is an extra local $Z_{N}$ symmetry which has to be spontaneously broken [4] under the way to the continuum limit. As we proposed in Ref. [0], if the large$N$ phase transition occurs for the Kazakov-Migdal model before the one separating the confinement and perturbative Higgs phases, one gets normal confinement (area law) in the intermediate region bounded by the two phase transitions. The problem is, however, that this scenario in not realized for the original model of Ref. [1].

The mean field analysis [5] indicates that no first order phase transition occurs, with decreasing mass parameter, before the gaussian model becomes unstable which is com-

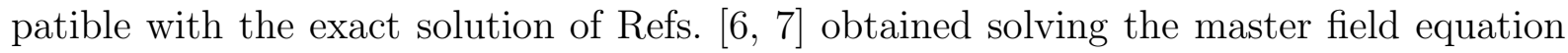
and reproduced by means of loop equations [8]. The instability is due to an unlimited condensation of scalar particles for an action which is unbounded from below and were lead to the Higgs phenomenon if a stabilizing self-interaction would be added.

The latter fact prompts the following way out. Let us consider fermions rather than bosons. On the one hand, the induced lattice action obtained by the large mass expansion looks for fermions very similar to that for scalars. On the other hand, since fermions never condense, the gaussian fermionic action in an external field is stable so one can arbitrarily decrease the mass parameter. Moreover, if one assumes that the gauge field is weakly fluctuating, the perturbative expansion for fermions looks like that for QCD and we expect, therefore, a phase transition which separates the perturbative region from the strong coupling phase with unbroken $Z_{N}$ symmetry and associated local confinement. This phase transition is to be identified with the large- $N$ phase transition discussed above.

In the present paper we propose to induce QCD a la Kazakov-Migdal by fermions in the adjoint representation of the gauge group on the lattice. We consider various types of lattice fermions: chiral, Kogut-Susskind and Wilson ones. We discuss first the adjoint scalar and fermionic models at large number of flavors $N_{f}$ and show they induce a single-plaquette lattice gauge theory quite similarly to Refs. [2, 3] for the fundamental representation. Using the mean field method we show that a first order large- $N$ phase transition occurs for chiral and Kogut-Susskind fermions at $N_{f}=1$. We conclude, therefore, these two models induce QCD. The similar conclusion presumably holds for Wilson fermions while the results for $N_{f}=1$ are less certain. We find an exact strong coupling 
solution of loop equations for the adjoint fermion model and show that for chiral fermions it is quite similar to the solution [0] for the Kazakov-Migdal model with the quadratic potential while for Wilson fermions the exact result coincides with the leading order of the large mass expansion. We point out that, as usual, the strong coupling solution is not sensitive to the first order large- $N$ phase transition which occurs inside the region where the large mass expansion is convergent. We discuss the possibility for the proposed models to be solvable as $N \rightarrow \infty$ in the weak coupling region where the Wilson loop averages obey normal area law.

\section{Inducing single-plaquette action at large $N_{f}$}

The scenario of breaking the $Z_{N}$ discussed in Section 1 can be completely realized at large number of flavors $N_{f}$ when the induced gauge theory is in our case the singleplaquette lattice gauge theory with the adjoint action quite similarly to Refs. [2, 3] for the fundamental representation of $S U\left(N_{c}\right)$. The large- $N$ phase transition occurs for the former action according to the pattern we proposed long time ago [9].

\subsection{Scalars}

For simplicity let us begin with the case of scalars. The extension of the Kazakov-Migdal model [1] to the case of $N_{f}$ flavors reads

$$
Z=\int \prod_{x, \mu} d U_{\mu}(x) \prod_{x} \prod_{f=1}^{N_{f}} d \Phi_{f}(x) \mathrm{e}^{\sum_{f=1}^{N_{f}} \sum_{x} N_{c} \operatorname{tr}\left(-m_{0}^{2} \Phi_{f}^{2}(x)+\sum_{\mu} \Phi_{f}(x) U_{\mu}(x) \Phi_{f}(x+\mu) U_{\mu}^{\dagger}(x)\right)}
$$

where $N_{f}$ fields $\Phi_{i}(x)\left(i=1, \ldots, N_{f}\right)$ take values in the adjoint representation of the gauge group $S U\left(N_{c}\right)$ and the link variable $U_{\mu}(x)$ belongs to the gauge group. For the purposes of the present paper, we restrict ourselves by the quadratic potential. The original Kazakov-Migdal model corresponds to $N_{f}=1$.

Calculating $N_{f}$ gaussian integrals, one rewrites the model (2.1) as the lattice gauge theory

$$
Z=\int \prod_{x, \mu} d U_{\mu}(x) \mathrm{e}^{-S_{i n d}\left[U_{\mu}(x)\right]},
$$

with the induced action given by the following large mass expansion

$$
S_{\text {ind }}[U]=-\frac{N_{f}}{2} \sum_{\Gamma} \frac{|\operatorname{tr} U(\Gamma)|^{2}}{l(\Gamma) m_{0}^{2 l(\Gamma)}} .
$$

For $N_{f}=1$ one recovers the result of Ref. [1].

We apply now the idea by Bander [2] of how to obtain the single-plaquette lattice action from the sum over loops on the r.h.s. of Eq. (2.3). Let $N_{f}$ amd $m_{0}^{2}$ simultaneously 
tend to $\infty$ so that $m_{0}^{2} \sim\left(N_{f}\right)^{\frac{1}{4}}$. Then the only single-plaquette term survives while those

associated with longer loops are suppressed at least as $\left(N_{f}\right)^{-\frac{1}{2}}$. Therefore we have shown in this limit that

$$
S_{\text {ind }}=-\frac{N_{f}}{8 m_{0}^{8}} \sum_{p}|\operatorname{tr} U(\partial p)|^{2}
$$

where $\partial p$ is the boundary of the plaquette $p$. In other words the induced theory is just the single-plaquette lattice gauge theory with the adjoint action.

As is known [9] the lattice gauge theory with the action (2.4) undergoes the (first order) large- $N$ phase transition at $N_{f} /\left(4 m_{0}^{8}\right) \approx 2$. This is exactly the phase transition

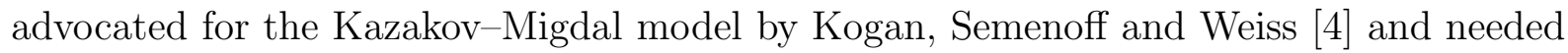
to induce QCD according to the scenario of Ref. [5]. Below the phase transition, the adjoint action (2.4) is reduced [9] at $N_{c}=\infty$ to a fundamental single-plaquette action and the Wilson loop averages obey area law.

It is crucial for this consideration that the critical value

$$
m_{*}^{2} \approx\left(\frac{N_{f}}{8}\right)^{\frac{1}{4}}>D
$$

as $N_{f} \rightarrow \infty$ so that the gaussian model (2.1) is stable. A mechanism to escape the instability while having the large- $N$ phase transition is pretty simple: the contribution of long loops in the effective action (2.3) which were lead to condensation is now suppressed by construction. This pattern looks like the idea to avoid instability by means of fermions since in the fermionic case the long loops do not condense due to alternating signs in the sum (see Eq. (2.9) below).

On the contrary there is no such phase transition for $m_{0}^{2}>D$ at $N_{f}=1$. This statement relies on the mean field study of Ref. [5] and is compatible with the exact solutions [7, 8]. Therefore, we expect the large- $N$ phase transition to occur for the model (2.1) only for $N_{f}$ larger than some value $N_{f *}$. This value is estimated in Section 3.5 by the use of the mean field method.

\subsection{Fermions}

Similarly to how Hamber [3] used large- $N_{f}$ limit to induce the Wilson single-plaquette lattice gauge theory by fermions in the fundamental representation of $S U\left(N_{c}\right)$, we can extend the results of the previous section to the fermionic case.

Let us consider the adjoint fermion model (AFM) which is defined by the partition function

$$
Z_{A F M}=\int \prod_{x, \mu} d U_{\mu}(x) \prod_{x} \prod_{f=1}^{N_{f}} d \Psi_{f}(x) d \bar{\Psi}_{f}(x) \mathrm{e}^{-S_{F}[\Psi, \bar{\Psi}, U]}
$$


Here $S_{F}[\Psi, \bar{\Psi}, U]$ is the standard action for fermions on the lattice:

$$
\begin{aligned}
& S_{F}[\Psi, \bar{\Psi}, U]=\sum_{f=1}^{N_{f}} \sum_{x} N_{c} \operatorname{tr}\left(m \bar{\Psi}_{f}(x) \Psi_{f}(x)\right. \\
& \left.-\sum_{\mu=1}^{D}\left[\bar{\Psi}_{f}(x) P_{\mu}^{-} U_{\mu}(x) \Psi_{f}(x+\mu) U_{\mu}^{\dagger}(x)+\bar{\Psi}_{f}(x+\mu) P_{\mu}^{+} U_{\mu}^{\dagger}(x) \Psi_{f}(x) U_{\mu}(x)\right]\right)
\end{aligned}
$$

where $\Psi_{f}(x)$ is the Grassmann anticommuting field while

$$
P_{\mu}^{ \pm}=r \pm \gamma_{\mu}
$$

stand for the projectors. The case $r=0$ corresponds to chiral fermions while $r=1$ is associated with Wilson fermions. As is well known, the chiral fermions describe $2^{D} N_{f}$ flavors in the naive continuum limit while Wilson fermions are associated with $N_{f}$ flavors.

The path integral over $\Psi$ can be calculated by the large mass expansion similarly to the scalar case. One gets for the induced action

$$
S_{\text {ind }}[U]=-N_{f} \sum_{\Gamma} \frac{|\operatorname{tr} U(\Gamma)|^{2}}{l(\Gamma) m^{l(\Gamma)}} \operatorname{Sp} \prod_{l \in \Gamma} P_{\mu}
$$

where Sp stands for the trace over the spinor indices of the path-ordered product of the projectors $P_{\mu}^{ \pm}$(plus or minus depends on the orientation of the link $l$ ) along the loop $\Gamma$.

The contribution of leading order in $1 / m$ comes from a plaquette which gives

$$
S_{\text {ind }}=-\frac{2^{\frac{D}{2}} N_{f}\left(1+2 r^{2}-r^{4}\right)}{m^{4}} \sum_{p}|\operatorname{tr} U(\partial p)|^{2} .
$$

If $m \sim N_{f}^{\frac{1}{4}}$, only this term survives in the large- $N_{f}$ limit while the others are suppressed at least as $N_{f}^{-\frac{1}{2}}$ similarly to the scalar case.

The estimate of the critical values of $m$ which are associated with the large- $N$ phase transition gives $m_{*} \approx\left(2 N_{f}\right)^{\frac{1}{4}}$ for chiral fermions and $m_{*} \approx\left(4 N_{f}\right)^{\frac{1}{4}}$ for Wilson fermions. As we shall see in the next section, the mean field analysis indicates that the phase transition certainly exists in the former case at $N_{f}=1$ as well.

\section{Mean field analysis}

While we have shown in the previous section that both adjoint scalar and adjoint fermion models induce the single-plaquette Wilson lattice gauge theory in the limit of large $N_{f}$, there is no hope for this limit to be solvable even at $N_{c}=\infty$ (this were correspond to an exact solution of Wilson lattice gauge theory at $N_{c}=\infty$ ). We investigate in this section AFM with $N_{f}=1$ which, as is discussed in Section 5, could be as solvable as the Kazakov-Migdal model. 


\subsection{Mean field with fermions}

We apply to AFM defined by the partition function (2.6) at $N_{f}=1$ the (variational) mean field approach of Ref. [5]. Since we substitute the mean field solely for the gauge field and exactly integrate over fermions, we expect the mean field method is applicable for our case despite the fermionic system.

Substituting the mean field value $\left[U_{\mu}(x)\right]_{i j}=\eta \delta_{i j}$ for all links of the lattice except the given one, we get the following self-consistency condition

$$
2\left(\eta-\eta^{2}\right)=\frac{1}{b_{A}}
$$

where

$$
b_{A}=\frac{\int \prod_{x} d \Psi(x) d \bar{\Psi}(x) \mathrm{e}^{-S_{F}[\Psi, \bar{\Psi}, \eta]} \frac{1}{N} \operatorname{tr}\left(\bar{\Psi}(0) P_{\mu}^{-} \Psi(0+\mu)+\bar{\Psi}(0+\mu) P_{\mu}^{+} \Psi(0)\right)}{\int \prod_{x} d \Psi(x) d \bar{\Psi}(x) \mathrm{e}^{-S_{F}[\Psi, \bar{\Psi}, \eta]}}
$$

and

$$
\begin{aligned}
& S_{F}[\Psi, \bar{\Psi}, \eta]=\sum_{x} N \operatorname{tr}(m \bar{\Psi}(x) \Psi(x) \\
& \left.-\eta^{2} \sum_{\mu=1}^{D}\left[\bar{\Psi}(x) P_{\mu}^{-} \Psi(x+\mu)+\bar{\Psi}(x+\mu) P_{\mu}^{+} \Psi(x)\right]\right) .
\end{aligned}
$$

Eq. (3.1) holds providing $\eta>1 / 2$, which is prescribed by the requirement for the corresponding one-matrix model to possess a weak coupling solution. To find the location of the phase transition one uses either geometric criterion which is based on terminating the $\eta \neq 0$ solution with increasing $m$ or the thermodynamic criterion which says that the phase transition occurs when free energies of the $\eta=0$ and $\eta \neq 0$ phases coincide.

\subsection{Chiral fermions}

For chiral fermions the gaussian integral on the r.h.s. of Eq. (3.2) can easily be calculated by the standard lattice technique (see Ref. [10]) to give

$$
b_{A}=\frac{2^{D}}{\eta^{2}} \prod_{\mu=1}^{D} \int_{-\pi}^{\pi} \frac{d p_{\mu}}{2 \pi} \frac{1-\frac{1}{D} \sum_{\mu} \cos p_{m}}{\frac{m^{2}}{2 \eta^{4}}+D-\sum_{\mu} \cos p_{\mu}}=\frac{2^{D}}{D \eta^{2}}\left(1-\frac{m^{2}}{2 \eta^{4}} \int_{0}^{\infty} d \alpha \mathrm{e}^{-\left(\frac{m^{2}}{2 \eta^{4}}+D\right) \alpha} \mathrm{I}_{0}^{D}(\alpha)\right) .
$$

Here $\mathrm{I}_{0}(\alpha)$ is the modified Bessel function.

The r.h.s. of Eq. (3.4) multiplied by $\eta^{2}$ monotonically increases with decreasing $m$ approaching the maximal value $2^{D} / D$ at $m=0$. Moreover, it can be approximated with great accuracy by the first term of the expansion in $x=1 /\left(\frac{m^{2}}{2 \eta^{4}}+D\right)$ which can be seen from the series expansion

$$
\begin{aligned}
\eta^{2} b_{A}=16 x-8 x^{2} & +32 x^{3}-42 x^{4}+168 x^{5}-320 x^{6}+1280 x^{7}-\frac{23765}{8} x^{8}+\frac{23765}{2} x^{9} \\
& -\frac{124047}{4} x^{10}+124047 x^{11}-\frac{1397319}{4} x^{12}+1397319 x^{13}+\mathcal{O}\left(x^{14}\right) .
\end{aligned}
$$


Thus, we substitute

$$
\frac{1}{b_{A}} \approx 2^{-D}\left(\frac{m^{2}}{2 \eta^{4}}+D\right) \eta^{2}
$$

and there is no need in numerical calculations.

The critical value $m_{*}$ can be easily determined from the above mentioned geometric criterion which says

$$
\left.\frac{\partial \eta}{\partial m}\right|_{*}=\infty \quad \text { or }\left.\quad \frac{\partial m}{\partial \eta}\right|_{*}=0
$$

at the critical point $m_{*}$. Differentiating Eq. (3.1) with the r.h.s. substituted by Eq. (3.6), one gets the solution

$$
m_{*} \approx 6.5, \quad \text { and } \quad \eta_{*} \approx \frac{2}{3}
$$

at $D=4$.

Some comments about the obtained critical values of the large- $N$ phase transitions in the case of chiral fermions are in order.

- In contrast to the scalar case analyzed in Ref. [5], the integral on the r.h.s. of Eq. (3.4) never diverges for $0<m^{2}<\infty$ which is a consequence of the stability of the fermionic system discussed above. Since $\eta^{2} b_{A}$ can now reach values larger than 0.5 , Eq. (3.1) always possesses a solution.

- The coefficient $2^{D}$ on the r.h.s. of Eq. (3.4) is nothing but the number of flavors which is associated with the naive continuum limit of chiral lattice fermions. Therefore, one could relate the appearance of the large- $N$ phase transition with an effective number of flavors similarly to Section 2.2. This point of view explains why, as is shown below, the large- $N$ phase transition still certainly occurs for Kogut-Susskind fermions describing $2^{\frac{D}{2}}$ flavors and presumably for Wilson lattice fermions which correspond to 1 flavor in the naive continuum limit.

\subsection{Kogut-Susskind fermions}

As is well known, the number of continuum flavors described by chiral fermions can be reduced by $2^{\frac{D}{2}}$ passing to Kogut-Susskind fermions. To this aim one performs the following transformation of the spinor $\Phi(x)$

$$
\Psi(x)=\left(\gamma_{1}\right)^{x_{1}}\left(\gamma_{2}\right)^{x_{2}} \ldots\left(\gamma_{D}\right)^{x_{D}} \chi(x)
$$

where the coordinates of the lattice vector $x_{\mu}$ are measured in the lattice units. In the new variables the fermionic lattice action (2.7) reads

$$
\begin{aligned}
& S_{F}[\chi, \bar{\chi}, U]=\sum_{x} N_{c} \operatorname{tr}(m \bar{\chi}(x) \chi(x) \\
& \left.-\sum_{\mu=1}^{D} \eta_{\mu}(x)\left[\bar{\chi}(x) U_{\mu}(x) \chi(x+\mu) U_{\mu}^{\dagger}(x)-\bar{\chi}(x+\mu) U_{\mu}^{\dagger}(x) \chi(x) U_{\mu}(x)\right]\right)
\end{aligned}
$$


where

$$
\eta_{1}(x)=1 ; \quad \eta_{\mu}(x)=(-1)^{x_{1}+\ldots+x_{\mu-1}} .
$$

Since the action (3.10) is diagonal w.r.t. spinor indices, one can keep only one component of $\chi(x)$ so that it can be considered as a scalar Grassmann variable. The normal spinors are reproduced in the continuum limit by $2^{D} \chi^{\prime}$ s taken at neighbor sites of the lattice. Such a procedure manifestly reduces the number of flavors in the continuum by $2^{\frac{D}{2}}$.

The mean field analysis of AFM with Kogut-Susskind fermions is quite similar to that of the previous section. One should only replace $2^{D}$ in Eqs. (3.4) end (3.6) by $2^{\frac{D}{2}}$ so that the self-consistency condition (3.1) at $D=4$ reads

$$
2\left(\frac{1}{\eta}-1\right)=\frac{m^{2}}{8 \eta^{4}}+1
$$

This equation determines the critical values associated with the large- $N$ phase transition to be

$$
m_{*} \approx 0.7, \quad \text { and } \quad \eta_{*} \approx \frac{1}{2} .
$$

Therefore, we have shown that the large- $N$ phase transition occurs for AFM with Kogut-Susskind fermions. The critical values (3.13) are smaller than those given by Eq. (3.8) and are near the limit of applicability.

\subsection{Wilson fermions}

For AFM with Wilson fermions $b_{A}$ is given by Eq. (3.2). The standard calculation [10] of the gaussian fermionic integral yields

$$
b_{A}=\frac{2^{\frac{D}{2}}}{D \eta^{2}} \prod_{\mu=1}^{D} \int_{-\pi}^{\pi} \frac{d p_{\mu}}{2 \pi}\left(1-\frac{m}{2 \eta^{2}} \frac{\frac{m}{2 \eta^{2}}-\sum_{\mu} \cos p_{\mu}}{\left(\frac{m}{2 \eta^{2}}-\sum_{\mu} \cos p_{\mu}\right)^{2}+\sum_{\mu} \sin ^{2} p_{\mu}}\right) .
$$

The integral on the r.h.s. of Eq. (3.14) possesses a number of interesting properties. Its large mass expansion

$$
\eta^{2} b_{A}=6\left(\frac{2 \eta^{2}}{m}\right)^{4}+66\left(\frac{2 \eta^{2}}{m}\right)^{6}+\mathcal{O}\left(\left(\frac{2 \eta^{2}}{m}\right)^{8}\right)
$$

begins with the term $\sim m^{-4}$ rather than $m^{-2}$ as for chiral fermions or bosons. The point is that backtracking paths in the sum over paths associated with $b_{A}$ are now forbidden since the product of projectors

$$
P_{\mu}^{+} P_{\mu}^{-}=r^{2}-1
$$

vanishes for $r=1$. For this reason our mean field coincides for Wilson fermions with the one which would be obtained directly from the induced action (2.9) where the backtracking paths never contribute due to unitarity of $U$. A price for this nice property is the complicated integral on the r.h.s. of Eq. (3.14). 
Since $b_{A}$ varies between 0 at $m=\infty$ and 1 at $m=0$, one expects Eqs. (3.1) and (3.7) to possess a solution for the critical value $m_{*}$. We performed an approximate numerical analysis and found $m_{*} \approx \eta_{*} \approx 0.5$ which looks like the case of Kogut-Susskind fermions. However, now an uncertainty of the results is bigger so that our conclusion is that, while the large- $N$ phase transition presumably occurs for Wilson fermions, more accurate investigation maybe by other methods is needed. However, the large- $N$ phase transition certainly occurs for AFM with Wilson fermions for $N_{f} \geq 2$.

\section{$3.5 N_{f}$ scalars}

The mean field method can be applied to estimate the critical value $N_{f *}$ below which the large- $N$ phase transition discussed in Section 2.1 disappears. The corresponding selfconsistency condition is given by Eq. (3.1) where $b_{A}$ reads

$$
b_{A}=\frac{N_{f}}{2 \eta^{2}} \int_{0}^{\infty} d \alpha \mathrm{e}^{-\frac{m_{0}^{2}}{\eta^{2}} \alpha} \mathrm{I}_{0}^{D-1}(\alpha) \mathrm{I}_{1}(\alpha)
$$

and $\mathrm{I}(\alpha)$ being the modified Bessel function.

The condition $\eta>1 / 2$, which is prescribed by the requirement for the corresponding one-matrix model to possess a weak coupling solution, yields $\eta^{2} b_{A}>1 / 2$ or $N_{f *}>30-$ a pretty big value - since the maximal value of the integral on the r.h.s. of Eq. (3.17) is 0.06 at $D=4$.

\section{Loop averages at strong coupling}

AFM can be exactly solved at $N_{c}=\infty$ in the strong coupling limit (i.e. before the large- $N$ phase transition). We obtain the result solving loop equations quite similarly to Ref. [8] where the Kazakov-Migdal model with the quadratic potential was solved by this method. The solution for AFM with chiral fermions obtained below is quite similar to that by Gross [7] for the Kazakov-Migdal model with the quadratic potential and coincides with the old results by Klugberg-Stern et al. [11] for the infinite-coupling limit of lattice $N_{c}=\infty$ QCD with chiral fermions. We show this fact is not a coincidence and is related to very peculiar properties of Wilson loop averages in AFM model as well as in the Kazakov-Migdal model. For Wilson fermions the exact solution of loop equations coincides for the same reason with the leading order of the large mass expansion. It will be explicitly demonstrated this strong coupling solutions are not singular, as usual, at the point of the first order large- $N$ phase transition which is determined by another criterion. 


\subsection{Loop equations in AFM}

All the observables in AFM can be expressed via the adjoint Wilson loop

$$
W_{A}(C)=\left\langle\frac{1}{N_{c}^{2}}\left(|\operatorname{tr} U(C)|^{2}-1\right)\right\rangle,
$$

where the average is understood with the same measure as in Eq. (2.6), and the fermionic path-dependent amplitude

$$
G_{i j}\left(C_{x y}\right)=\left\langle\frac{1}{N_{c}} \operatorname{tr}\left(\Psi_{i}(x) U\left(C_{x y}\right) \bar{\Psi}_{j}(y) U^{\dagger}\left(C_{x y}\right)\right)\right\rangle
$$

where $i$ and $j$ are spinor indices.

The amplitude $G_{i j}\left(C_{x y}\right)$ obeys two sets of loop equations which are analogous to the loop equations in QCD with quarks (in the fundamental representation of the gauge group). It is simple to obtain an equation resulting from the Dirac equation for $\Psi(x)$ which reads

$$
m G\left(C_{x y}\right)-\sum_{\mu=1}^{D}\left[P_{\mu}^{+} G\left(C_{(x+\mu) x} C_{x y}\right)+P_{\mu}^{-} G\left(C_{(x-\mu) x} C_{x y}\right)\right]=\delta_{x y} W_{A}\left(C_{x y}\right)
$$

where the matrix multiplication over spinor indices is implied. Here the path $C_{(x+\mu) x} C_{x x}$ is obtained by attaching the link $(x, \mu)$ to the path $C_{x x}$ at the end point $x$ as is depicted in Fig. 1. The projectors are given by Eq. (2.8) for chiral and Wilson fermions or by Eq. (3.11) for Kogut-Susskind fermions. Due to the presence of the delta-function, $W_{A}$ on the r.h.s. of Eq. (4.3) always enters for closed loops, i.e. it is an object of the type (4.1).

Similarly to Ref. [8] where an analog of Eq. (4.3) was solved for the Kazakov-Migdal model with the quadratic potential at strong coupling, Eq. (4.3) admits the ansatz

$$
G_{i j}\left(C_{x y}\right)=G_{L}\left(\prod_{l \in C_{x y}} P_{\mu}^{ \pm}\right)_{i j}
$$

where plus or minus corresponds to the direction of the link $l$ which belongs to the contour $C_{x y}$ and $L$ is the algebraic length [12] (i.e. the one after contracting the backtrackings) of $C_{x y}$. The spin factor is needed to cancel the projectors in Eq. (4.3).

For such an ansatz, Eq. (4.3) for AFM is reduced to the following recurrent relations on $G_{L}$

$$
\begin{aligned}
m G_{L}-G_{L-1}-(2 D-1) \sigma G_{L+1} & =0 \text { for } L \geq 1 \\
m G_{0}-2 D \sigma G_{1} & =1
\end{aligned}
$$

where $\sigma=r^{2}-1$. These equations are quite similar to the corresponding equations for the Kazakov-Migdal model with the quadratic potential which are given [8] by the same formulas with $\sigma=1$ and $m$ replaced by $2 m_{0}^{2}$. The drastic simplification of the loop 
equation (4.3) is due to the fact [5] that $W_{A}(C)$ vanishes in the strong coupling region at $N=\infty$ except for $C$ with vanishing minimal area $A_{\min }(C)$ (i.e. contractable to a point owing to unitarity of $U$ 's):

$$
W_{A}(C)=\delta_{0, A_{\min }(C)}+\mathcal{O}\left(\frac{1}{N^{2}}\right)
$$

Eq. (4.5) can be solved introducing the generating function

$$
G(\lambda)=\sum_{L=0}^{\infty} G_{L} \lambda^{L}
$$

The solution at $\sigma \neq 0$ (the solution for $\sigma=0$ i.e. for Wilson fermions is described in the next section) is expressed via $G_{0}$ which was up to now arbitrary. It can be determined imposing [8] the single-pole analytic structure of $G(\lambda)$ as a function of the spectral parameter $\lambda$. This requirement unambiguously determines $G(\lambda)$ to be

$$
G(\lambda)=\frac{2(2 D-1) \sigma G_{0}}{\left(\sqrt{m^{2}+4(1-2 D) \sigma}-m\right) \lambda+2(2 D-1) \sigma}
$$

and

$$
G_{0}=\frac{2 D-1}{m(D-1)+D \sqrt{m^{2}+4(1-2 D) \sigma}} .
$$

The single-pole prescription is justified in the next section.

\subsection{Properties of strong coupling solution}

Let us consider the strong coupling solution of AFM, obtained in the previous section, from the viewpoint of the path representation of the amplitude (4.2). At $N_{c}=\infty$ one gets

$$
G_{i j}\left(C_{x y}\right)=\sum_{\Gamma_{y x}} \frac{1}{m^{l(\Gamma)}} W_{A}\left(C_{x y} \Gamma_{y x}\right)\left(\prod_{l \in \Gamma_{y x}} P_{\mu}^{ \pm}\right)_{i j}
$$

where the path $C_{x y} \Gamma_{y x}$ is closed by construction. Eq. (4.10) is nothing but the standard large mass representation [10] of the amplitude in lattice gauge theories with fermions while the Wilson loop is taken in the same representation as fermions (adjoint in our case). The fact that the only one-loop average enters Eq. (4.10) is due to $N_{c}=\infty$ 12.

In the strong coupling region where $W_{A}(C)$ is given by the simple formula (4.6), the r.h.s. of Eq. (4.10) drastically simplifies. The path $\Gamma_{y x}$ must coincide with $C_{x y}$ passing in the opposite direction modulo backtrackings ${ }^{2}$ since $W_{A}$ equals 1 in this case and 0 otherwise. Therefore, the problem of calculating the amplitude $G$ by the sum over paths

\footnotetext{
${ }^{2}$ One should not confuse these backtrackings of $\Gamma$ with possible backtrackings of $C$ discussed above. The latter ones can be always contracted due to unitarity.
} 
is reduced to a problem of counting backtrackings of one-dimensional trees embedded in a $D$-dimensional hypercubic lattice. The above formula (4.8) represents the solution of this problem. Notice that since the spin structure on the r.h.s. of Eq. (4.10) factorizes, the coefficient functions $G_{L}$ are quite similar for chiral fermions and scalars.

We would like to point out that exactly the same problem emerges in lattice QCD at infinite coupling where quarks are in the fundamental representation but the Wilson loops for non-contractable paths vanish since the coupling is infinite. Its solution obtained for chiral fermions by Klugberg-Stern et al. [11 using a different method exactly coincides with Eq. (4.9).

The result of Ref. [11] was reproduced by Mkrtchyan and one of the authors [13] by means of loop equations. While the loop equation resulting from the Dirac equation for fermions looks like Eq. (4.3) with $W_{A}$ on the r.h.s. substituted by the fundamental Wilson loop, the loop equations resulting from the (lattice) Maxwell equation are simplified since fermions are now in the fundamental representation. All the definitions in this case are the same as above with the path ordered phase factor in the adjoint representation substituted by the one in the fundamental representation, say the definition of the fundamental amplitude $G$ reads

$$
G_{i j}^{F}\left(C_{x y}\right)=\left\langle\Psi_{i}^{F}(x) U\left(C_{x y}\right) \bar{\Psi}_{j}^{F}(y)\right\rangle .
$$

The equation resulting from the variation of $U_{\mu}(x)$ at the link $(x, \mu) \in C_{x y}$ emanating from the point $x$ reads

$$
G^{F}\left(C_{x y}\right)=G^{F}\left(C_{x(x+\mu)}\right) \gamma_{\mu} G^{F}\left(C_{x y}\right)-G^{F}(0) \gamma_{\mu} G^{F}\left(C_{(x+\mu) y}\right)
$$

for chiral fermions. For the ansatz (4.4) one gets

$$
G_{L}=G_{0} G_{L-1}-\sigma G_{1} G_{L} \quad \text { for } \quad L \geq 1 \text {. }
$$

While the corresponding loop equation for AFM does not coincide, generally speaking, with Eq. (4.12), for the ansatz (4.4) it should reduce, as is discussed above, to Eq. (4.13). At $L=1$ this equation gives one more relation between $G_{0}$ and $G_{1}$ which together with the $L=0$ equation (4.5) result in a quadratic equation for $G_{0}$ having an unambiguous solution (4.9) expandable in $1 / m$. Therefore, we have shown that the above strong coupling solution for AFM with chiral fermions (or for the Kazakov-Migdal model with the quadratic potential) is unique. In particular, we have justified the single-pole procedure of Ref. [8].

An analogous exact strong coupling solution of loop equations for AFM with Wilson fermions can be obtained as well. Since $\sigma=0$ for $r=1$, Eqs. (4.5) are further simplified so that the solution reads

$$
G_{L}=\frac{1}{m^{L}}
$$

\footnotetext{
${ }^{3}$ The bilinear in $G$ terms were missing in the original paper by Weingarten [14].
} 
which coincides with the leading order of the large mass expansion. The reason behind this it as follows. As is discussed in Section 3.4, the problem of backtrackings is missing for Wilson fermions due to the property (3.16) of projectors $P_{\mu}^{ \pm}$. Therefore, the leading term of the large mass expansion gives the exact result for AFM with Wilson fermions.

Some comments about the properties of the exact strong coupling solutions (4.8) and (4.14) of AFM with chiral and Wilson fermions are now in order:

- The solutions (4.8), (4.14) are not singular for $m^{2}>0$ in contrast to the scalar case. Therefore, the strong coupling solution is fermion case is stable everywhere. However, AFM undergoes the first order large- $N$ phase transition after which the strong coupling solution is not applicable. As usual for first order phase transition, this critical point is quite regular from the viewpoint of the large mass expansion and is determined by another method (like the mean field approach of Section 3). After the large- $N$ phase transition occurs, Eq. (4.6) that corresponds to local confinement is no longer valid and should be replaced by normal area law. The ansatz (4.4) is no longer applicable in this region and the solution of loop equations would be quite different.

- While for $D=1$ the strong coupling solution (4.8) coincides with the free theory amplitudes, it looks simpler for $D>1$ than the corresponding free theory results given by formulas which are similar to those of Section 3 .

- All the statements of Ref. [11] concerning the breaking of chiral symmetry for QCD with chiral fermions at infinite coupling are applicable to our case. In particular, no extra phase transition associated with spontaneous breaking of chiral symmetry occurs with decreasing mass and $\langle\bar{\Psi} \Psi>$ which is determined by Eq. (4.9) is non-vanishing at $m=0$.

- Loop equations turned out to be perfect to solve the backtracking problem arising in the large mass expansion and unambiguously determine the solution without any assumptions.

\section{Conclusions and discussion}

The main conclusion we draw from the results obtained in the present paper is that a simple replacement of scalars by fermions in the Kazakov-Migdal model cures the problem of instability and provides the large- $N$ phase transition when the fermion mass is decreased. While in the strong coupling phase (before the phase transition) one gets local confinement which is characterized by Eq. (4.6), normal area law holds in the weak coupling phase (after the phase transition). This phase resembles very much the standard lattice gauge theory. Moreover, at large- $N_{f}$ a single plaquette lattice action arises. Hence, we conclude that AFM induces QCD.

The obtained exact strong coupling solution of AFM does not know anything about the large- $N$ phase transitions which is associated with freezing of the gauge field $U_{\mu}(x)$ 
near some mean field value $\eta$ while $\eta=0$ in the strong coupling region. This is usual for first order phase transitions. The weak coupling solution of AFM should be quite different from the strong coupling one.

After a nonvanishing value of $\eta$ emerges, the arguments of Ref. [1] about the continuum limit caused by long loops become applicable. We expect therefore that AFM will induce continuum QCD as well. One more argument in favor of this is the mentioned above similarity between the weak coupling phases of AFM and of the Wilson lattice gauge theory.

A very interesting question is whether the solubility of the Kazakov-Migdal model by means of the master field equation [6] would be pursued for AFM. This were give a way to solve AFM in the weak coupling region which would be an alternative to loop equations. As is mentioned already, we do not think this would be the case for any $N_{f}$. We see however some hope to derive an extension of the Itzykson-Zuber-Metha integral, which played a crucial role in the approach of Refs. [1, 6], to the case of Kogut-Susskind like fermions:

$$
I[\psi, \chi]=\int d U \mathrm{e}^{N_{c} \operatorname{tr} \psi U \chi U^{\dagger}}
$$

where $\psi$ and $\chi$ are Grassmann variables which transform under the adjoint representation of $S U\left(N_{c}\right)$. While it is not clear of how to reduce $\psi$ and $\chi$ to a diagonal form, the integral over the Haar measure on the r.h.s. of Eq. (5.1) could be tractable by other methods. This problem deserves future investigations.

\section{Note added}

When this paper was being prepared for publication, there appeared two more papers [15, 16] on the Kazakov-Migdal model. While Ref. [15] deals mostly with the quadratic potential, a strong coupling solution for a general model with fundamental Wislon fermions is obtained by Migdal [16]. 


\section{References}

[1] V.A.Kazakov and A.A.Migdal, Induced QCD at large $N$, Paris / Princeton preprint LPTENS-92/15 / PUPT-1322 (June, 1992)

[2] M.Bander, Phys. Lett. 126B (1983) 463

[3] H.W.Hamber, Phys. Lett. 126B (1983) 471

[4] I.I.Kogan, G.W.Semenoff and N.Weiss, Induced $Q C D$ and hidden local $Z_{N}$ symmetry, UBC preprint UBCTP-92-022 (June, 1992)

[5] S.Khokhlachev and Yu.Makeenko, The problem of large- $N$ phase transition in KazakovMigdal model of induced QCD, Moscow preprint ITEP-YM-5-92 (July, 1992)

[6] A.A.Migdal, Exact solution of induced lattice gauge theory at large N, Princeton preprint PUPT-1323 (June, 1992); 1/N expansion and particle spectrum in induced QCD, Princeton preprint PUPT-1332 (July, 1992); Phase transitions in induced QCD, Paris preprint LPTENS-92/22 (August, 1992)

[7] D.Gross, Some remarks about induced QCD, Princeton preprint PUPT-1335 (August, 1992)

[8] Yu.Makeenko, Large- $N$ reduction, master field and loop equations in Kazakov-Migdal model, Moscow preprint ITEP-YM-6-92 (August, 1992)

[9] S.B.Khokhlachev and Yu.M.Makeenko, Phys. Lett. 101B (1981) 403; ZhETF 80 (1981) 448 (Sov. Phys. JETP 53 (1981) 228)

[10] K.G.Wilson, Erice lecture notes, 1975

[11] H.Klugberg-Stern, A.Morel, O.Napoly and B.Peterson, Nucl. Phys. B190 [FS3] (1981) 504

[12] A.A.Migdal, Phys. Rep. 102 (1983) 199

[13] S.Khokhlachev and R.Mkrtchyan, unpublished (1983)

[14] D.Weingarten, Phys. Lett. 87B (1979) 97

[15] I.I.Kogan, A.Morozov, G.W.Semenoff and N.Weiss, Continuum limits of 'induced $Q C D$ ': lessons of the gaussian model at $D=1$ and beyond, UBC preprint 92-27 (August, 1992)

[16] A.A.Migdal, Mixed model of induced QCD, Paris preprint LPTENS-92/23 (August, 1992) 


\section{Figures}

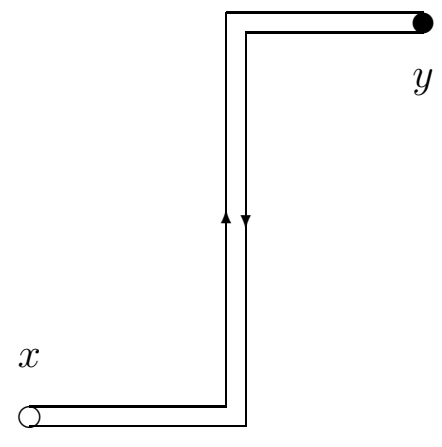

a)

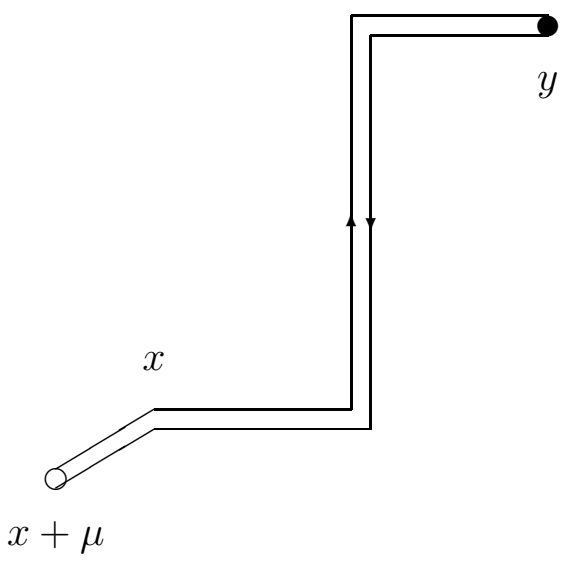

b)

Fig. 1 The graphic representation for $G_{i j}\left(C_{x y}\right)$ (a) and $G_{i j}\left(C_{(x+\mu) x} C_{x y}\right)$ (b) entering Eq. (4.3). The empty and filled circles represent $\Psi_{i}(x)\left(\right.$ or $\left.\Psi_{i}(x+\mu)\right)$ and $\bar{\Psi}_{j}(y)$, respectively. The oriented solid lines represent the path-ordered products $U\left(C_{x y}\right)$ and $U\left(C_{(x+\mu) x} C_{x y}\right)$. The color indices are contracted according to the arrows while the spinor ones are associated with circles. 